\title{
Excited state structural analysis for correlated many-electron systems
}

\author{
A.V. Luzanov \\ STC "Institute for Single Crystals", \\ National Academy of Sciences of Ukraine, 61001 Kharkiv, Ukraine
}

Received April 25, 2014

\begin{abstract}
The excited states structural analysis (ESSA) which was previously developed by the author and coll. $(1980,2010)$ is extended to a typical equation-of-motion approach within the coupled cluster theory. The extended ESSA allows one to interpret highly correlated excited states, particularly those which occur in molecular singlet fission processes and related optoelectronic devices.
\end{abstract}

Развитый ранее автором и сотр. $(1980,2010)$ структурный анализ возбужденных состояний расширен на случай метода уравнений движения применительно к теории связанных кластеров. Расширенная схема анализа позволяет интерпретировать сильнокоррелированные возбужденные состояния, возникающие в частности в молекулярных процессах синглетного расщепления и в соответствующих оптоэлектронных устройствах.

Структурний аналіз збуджених станів для корельованих багатоелектронних систем. А.В.Лузанов.

Структурний аналіз збуджених станів, що його раніше розвинуто автором та співр. $(1980,2010)$, поширено на випадок методу рівнянь руху стосовно теорії з'вязаних кластерів. Узагальнена схема аналізу дозволяє інтерпретацію сильнокорельованих збуджених станів, що виникають у молекулярних процесах синглетного розщеплення та відповідних оптоелектронних пристроях.

\section{Introduction}

Excited states analysis of complex molecular structures remains one of the principal topics of the molecular electronic structure theory and its multifarious applications. In particular, understanding of excited states and the microscopic theory of photoarrangements provides a conceptual framework for devising new materials from "first principles". These problems are of deep interest in the modern optoelectronics (OLEDs [1, 2], solar cells [3], multiple exciton generation in quantum dots [4], singlet fission processes [5-7], and many others).

Many years ago a practically useful approach was proposed [8] for analyzing the so-called CIS (singly excited configuration interaction) states. Our approach was termed the excited state structural analysis
(ESSA) [9, 10]. The key quantities of ESSA are the excitation indices $L_{A}^{*}$ and charge transfer numbers $l_{A \rightarrow B}$, giving together an intuitive chemical-like description of the CIS states.

The extension of the previous ESSA scheme [8] to the popular TDDFT method (the time-dependent density functional theory) was given in [9]. Nevertheless, CIS and TDDFT are not reliable models for highly correlated electronic states. Just such are the electronic states involved in the multiple exciton generation and singlet fission processes. Therefore, we need an extension of the ESSA scheme to multiconfigurational excited states other than too simplified CIS or DFT states. Unfortunately, the more advanced approaches to the excited state analysis (via one-particle transition density 
matrices) $[10,11]$ are seemingly not able to treat some important excited states which occur in multiple exciton generation processes. One of many-body approaches which effectively treat electron correlation in excited states is the EOM-CCSD scheme (equation-of-motion coupled-cluster singles and doubles method) [12]. A simplified EOMCCSD variant in the form of the so-called SAC-CI (symmetry adapted cluster/configuration interaction) method is just applicable to multiexciton states [4]. Thus the primary purpose of the present work is to provide the intuitive understanding of the appropriate sophisticated correlated methods by constructing suitable EOM-CCSD analogues of the above mentioned indices $L_{A}^{*}$ and $l_{A \rightarrow B}$.

\section{ESSA for single excited configuration interaction}

Following [8,9] we shortly recall the ESSA basic notions. Given a molecule containing fragments (molecular subunits) $A$, $B, \ldots, C$, let the state vector $|A B \ldots C\rangle$ (a formal reference product of the fragment wave functions) be a representation of the ground-state wave function. How to estimate a percent excitation character which can be assigned to each molecular fragment $A$ ? The corresponding quantity $L^{*}{ }_{A}$ (excitation index) can be introduced to give the probability of the excitation localization on the given fragment $A$. By construction, the full set $\left\{L_{A}^{*}\right\}$ is normalized to unity: $\sum_{A} L_{A}^{*}=1$.

One can further detail the analysis by distinguishing two excitation localization types. The first one is referred to as purely local excitations $\left|A^{*} B \ldots\right\rangle,\left|A B^{*} \ldots\right\rangle$ etc. (LE type), while the second one is referred to as charge transfer excitations $\left|A^{+} B^{-} \ldots\right\rangle,\left|A^{-} B^{+} \ldots\right\rangle$, etc. (CT type). With each LE type $\left|A^{*} B . ..\right\rangle$ we will relate the corresponding probability weight $l_{A}$ (partial excitation localization index), and with each CT type $\left|A^{+} B^{-} \ldots\right\rangle$ we will relate a weight $l_{A \rightarrow B}$ (charge transfer number, or CT number). It is natural to make the identification $l_{A \rightarrow A}=l_{A}$, and then the full probability set $\left\{l_{A \rightarrow B}\right\}$ should be also normalized to unity. Two sets, $\left\{L_{A}^{*}\right\}$ and $\left\{l_{A \rightarrow B}\right\}$, are interrelated as follows: $L_{A}^{*}=\sum_{B}\left(l_{A \rightarrow B}+l_{B \rightarrow A}\right) / 2$. For CIS wave functions, the CT numbers are computed very simply $[8,9,13]$.

\section{EOM - CCSD in a nutshell}

Before extending ESSA we recall the main features of the EOM-CCSD theory of excited states [12]. The key idea of the equation of motion method is to find a general type excitation operator $\hat{R}$, such that it will generate an excited state as follows: $\left|\Psi^{*}\right\rangle=\hat{R}\left|\Psi^{0}\right\rangle$ where $\left|\Psi^{0}\right\rangle$ is an exact or almost exact ground state wave function. It allows to study highly correlated states by taking into account most essential electronic degrees of freedom of $\hat{R}[12,14]$. Really, $\hat{R}$ can be generally decomposed into components including the most important one-body $R_{1}$ and two-body $\hat{R}_{2}$ excitation operators. In practice $\left|\Psi^{*}\right\rangle$ is taken as the CCSD ground state vector, so $\left|\Psi^{*}\right\rangle=$ $\left(\hat{R}_{1}+\hat{R}_{2}\right)\left|\Psi^{0}\right\rangle$. Operators $\hat{R}_{1}$ and $\hat{R}_{2}$ can be formed as the usual one-electron and twoelectron excitation operators:

$$
\begin{gathered}
\hat{R}_{1}=\sum_{i}^{o c c} \sum_{a}^{v a c} r_{a i} a_{a}^{+} a_{i}, \\
\hat{R}_{2}=\frac{1}{2} \sum_{i j}^{o c c} \sum_{a b}^{v a c} r_{a b, i j} a_{a}^{+} a_{b}^{+} a_{j} a_{i} .
\end{gathered}
$$

Here $a_{a}^{+}$is a creation operator for vacant (virtual) spin-orbital $\left|\varphi_{a}\right\rangle, a_{i}$ is an annihilation operator for occupied spin-orbital $\left|\varphi_{i}\right\rangle$, and $r_{a i}, r_{a b, i j}$ are corresponding excitation (particle-hole) amplitudes. Then the special EOM-CCSD secular problem is formulated for amplitudes $r_{a i}, r_{a b, i j}$. However, in the EOM-CCSD approach the Hamiltonian matrix is non-Hermitian, and the corresponding eigenvectors obey the specific normalization condition: $\sum_{a, i} l_{a i} r_{a i}+\sum_{a b, i j} l_{a b, i j} r_{a b, i j}=1$ where $l_{a i}$, $l_{a b, i j}$ are components of the left eigenvector corresponding to the right eigen vector with components $r_{a i}, r_{a b, i j}$.

\section{ESSA for EOM-CCSD}

Now we proceed to our main problem: how to define CT numbers at the EOM-CC level. As in the case of CIS states we must transform excitation amplitudes $r_{a i}, r_{a b, i j}$, etc. to an orthonormal atomic spin-orbital basis $\left\{\left|\chi_{\mu}\right\rangle\right\}$. This customary procedure gives us atomic amplitudes $r_{\mu, v}, r_{\mu \nu, \rho \sigma}$ etc., and in these notations the normalization is equivalent to the equation 


$$
\sum_{\mu \nu} l_{\mu \nu} r_{\mu \nu}+\sum_{\mu \nu \rho \sigma} l_{\mu \nu, \rho \sigma} r_{\mu \nu, \rho \sigma}=1 .
$$

The individual contributions here can be analyzed in the same manner as similar atomic terms $\left(\tau_{\mu \nu}\right)^{2}$ in the CIS theory $[8,9]$. Indeed, in practice the right and left vector components, such as $r_{\mu \nu}$ and $l_{\mu v}$, turn out to be sufficiently close each other. Thus, terms $l_{\mu \mu} r_{\mu \mu} \cong r_{\mu \mu}^{2}$ allow the same interpretation as purely local excitation indices $\left(\tau_{\mu \mu}\right)^{2}=l_{\mu \rightarrow \mu}$ in $[8,9]$. Analogously, the other terms in the probability sum (2) can be naturally characterized in terms of LE and CT states.

For definiteness, below we present the charge transfer analysis for a two-fragment molecule $\mathrm{AB}$. The required additional information can be extracted from atomic two-electron excitation amplitudes $r_{\mu \nu, \rho \sigma}$ and $l_{\mu \nu, \rho \sigma}$. We recall that the CIS atomic amplitude $\tau_{\mu \nu}$ has a meaning of the probability amplitude for transferring single electron from $\left|\chi_{v}\right\rangle$ to $\left|\chi_{\mu}\right\rangle$. In the same manner, EOMCCSD atomic amplitudes can be interpreted. For instance, if $\mu, \nu, \rho, \sigma \in A$, then the quantities $w_{\mu, \nu}$ $\equiv l_{\mu, v} r_{\mu, \nu}$ and $w_{\mu \nu, \rho \sigma} \equiv l_{\mu \nu, \rho \sigma} r_{\mu \nu, \rho \sigma}$ contribute to the purely local excitation $\mid A^{*} B$... $\rangle$. As a result, we obtain the partial localization index $l_{A}$ at the EOM-CCSD level:

$$
l_{A}=W^{I}\left(A^{*}\right)+W^{I I}\left(A^{* *}\right),
$$

where

$$
\begin{aligned}
W^{I}\left(A^{*}\right) & =\sum_{\mu \nu}^{(A)} w_{\mu, \nu}, \\
W^{I I}\left(A^{* *}\right) & =\sum_{\mu \nu \rho \sigma}^{(A)} w_{\mu, \nu, \rho \sigma} .
\end{aligned}
$$

Analogously, $l_{B}$ is defined: $l_{B}=W^{\mathrm{I}}\left(B^{*}\right)+$ $W^{\mathrm{II}}\left(B^{* * *}\right)$. The EOM-CCSD charge transfer numbers can be computed in terms of individual probabilities $w_{\mu \nu, \rho \sigma}$, as follows:

$$
\begin{gathered}
l_{A \rightarrow B}=W^{I}\left(A^{+} B^{-}\right)+W^{I I}\left(A^{*+} B^{-}\right)+ \\
+W^{I I}\left(A^{+} B^{*-}\right)+W^{I I}\left(A^{++} B^{--}\right), \\
W^{I}\left(A^{+} B^{-}\right)=\sum_{\mu} \sum_{v} w_{v, \mu}, \\
W^{I I}\left(A^{*+} B^{-}\right)= \\
\qquad \sum_{\mu \rho \sigma} \sum_{v}(B)
\end{gathered}
$$

Functional materials, 21, 2, 2014
(A) $(B)$

$$
\begin{aligned}
W^{I I}\left(A^{+} B^{*-}\right) & =2 \sum_{\mu} \sum_{v \rho \sigma} w_{v \sigma, \mu \rho} \\
W^{I I}\left(A^{++} B^{--}\right) & =\sum_{\mu \rho}^{(A)} \sum_{v \sigma}^{(B)} w_{v \sigma, \mu \rho}
\end{aligned}
$$

Evidently, $l_{B \rightarrow A}$ is produced by interchanging $A$ and $B$ in (5).

The rest term from the probability sum (2) is the index

$$
l_{A B}^{* *}=4 \sum_{\mu \rho}^{(A)} \sum_{\nu \sigma}^{(B)} w_{\mu \nu, \rho \sigma} .
$$

This is an additional localization quantity which could not appear in the CIS-type oversimplistic schemes because $l_{A B}^{* *}$ corresponds to contributions from biexcited states $\left|A^{*} B^{*} \ldots\right\rangle$ (analogues of biexcitons in the solid state optics). Finaly, consistent with all these relations, we define the EOMCCSD excitation index $L^{*}{ }_{A}$ as follows:

$$
L_{A}^{*}=l_{A}+\left(l_{A \rightarrow B}+l_{B \rightarrow A}+l_{A B}^{* *}\right) / 2 .
$$

The analysis of a many-fragment molecule AB..CD is both complicated and enriched by extra types of excitations such as $\left|A^{+} B^{-} C^{*} \ldots\right\rangle$ and $\left|A^{+} B^{-} C^{+} D^{-} \ldots\right\rangle$. The ESSA full account for this general case will be given elsewhere. Notice that it is possible that for highly excited states some indices, e. g., $W^{\mathrm{II}}\left(A^{++} B^{--}\right)$, can be negative, yet small, values. In this case one may use the absolute index values or pass to the Hermitized EOM-CCSD theory.

\section{Application and discussion}

We will illustrate the given extended ESSA scheme by two simplest examples (see Table). The results for three lowest-lying electronic transitions of both systems are presented. Each state is characterized by the ESSA diagram, the double excitation weight (6), $l_{A B}^{* *}$, in $\%$, and the collectivity number $\kappa[8,9]$. In the ESSA diagrams we display, in $\%$, the fragment excitation indices $L_{A}^{*}$ (at the fragment center or near the fragment) and the corresponding CT numbers $l_{A \rightarrow B}$ (at the arrows).

Our first example is the calicene (triapentafulvalene) representing a rather typical conjugated $\pi$-system which has been studied frequently within $\pi$-approximation even in the modern literature (see [15] and references therein). The calicene $\pi$-system is con- 
Table. ESSA diagrams (all indices in \%), double excitation contributions $l_{A B}^{* *}$ (in \%), and transition collectivity indices $\kappa$ for three lowest-lying $\pi \rightarrow \pi^{*}$ calicene transitions (the left panel) and $\sigma \rightarrow \sigma^{*}$ transitions in the hydrogen dimer (the right panel).

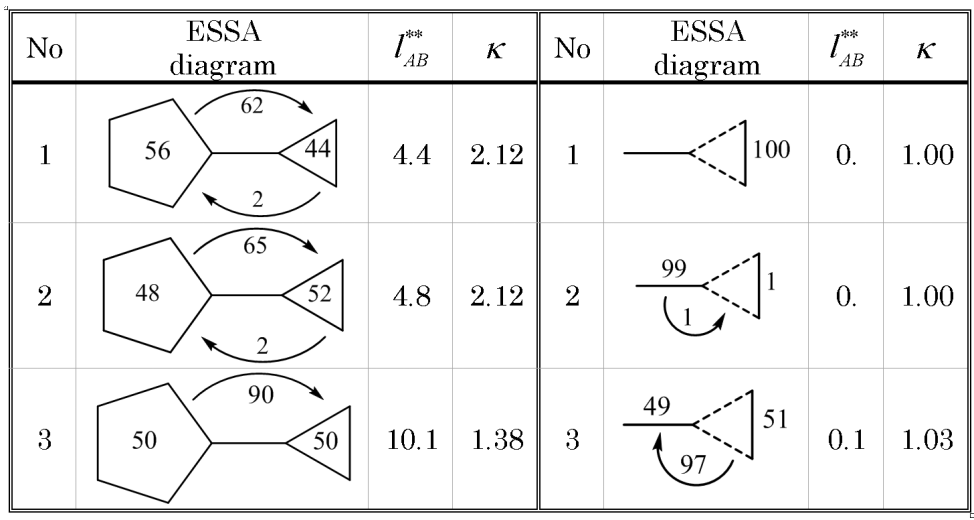

sidered here by applying the conventional Pariser-Parr-Pople model. The corresponding fragments are the five- and three-membered carbon rings. We see that the lowest $\pi \pi^{*}$ excitations are assigned to transitions involving a strong CT. From the direction of this CT we also observe that the calicene lowest excited states become less aromatic that in the ground state. It is essential that this assignment is obtained in the framework of the high-level electron correlation (EOM-CCSD) theory (see the $l_{A B}^{* *}$ values in the Table). Incidentally, the transitions are of a small collectivity. Notice that collectivity numbers turn out to be quite important physically for detecting plasmon-like excitations in large molecular systems [16].

The second example is the hydrogen molecule dimer $\left(\mathrm{H}_{2}\right)_{2}$. For the dimer we used the observed T-shaped geometry and carried out the $a b$ initio EOM-CCSD scheme employing the standard 6-31G basis set. From the Table we observe that two lowest-lying transitions are fully localized on corresponding individual $\mathrm{H}_{2}$ molecules, whereas the third transition is delocalized and of almost pure CT character. These excitations are very similar to the related CIS states. In addition, however, EOM-CCSD also allows us to produce a highly correlated excited state (the fifth one which was not included in the Table). The state turns out to be predominantly biexcited in its nature $\left(l_{A B}^{* *}=98 \%\right)$. More then that, this state has a triplet-triplet (TT) character. It is just the kind of molecular excitation which, with some additional requirements, permits the remarkable singlet fission photo process (a fission of the singlet excitation into pairs of triplet ones) [6]. In our example the above assignment to TT-transition is confirmed by direct calculations of local squared spins (the computational scheme [17] is used). Namely, spin-spin autocorrelators $\left\langle\mathbf{S}_{A} \cdot \mathbf{S}_{A}\right\rangle=$ $\left\langle\mathbf{S}_{B} \cdot \mathbf{S}_{B}\right\rangle=1.95$, that is, in the dimer each hydrogen molecule is in fact a triplet species.

On this account we suggest that EOMCCSD, SAC-CI and related models have yet untapped potential for studies of specifically entangled spins occurring in singlet fission and related photovoltaic phenomena $[7,2]$. Previously, such a possibility was not expected based on few unfavorable examples in EOM-CCSD applications [18]. At any rate, additional studies including the proposed ESSA approach for analyzing the EOM-CCSD excited states are worth undertaking.

\section{References}

1. Organic Light-emitting Diodes: Materials, Devices and Applications, ed. by A.Buckley, Woodhead Publishing, Cambridge (2013).

2. B.Minaev, G.Baryshnikov, H.Agren, Phys. Chem. Chem. Phys., 17, 1719 (2014).

3. Organic Photovoltaics. Concepts and Realization, ed. by C.J.Brabec, V.Dyakonov, J.Parisi, N.S.Sariciftci, Springer, Berlin (2003).

4. H.M.Jaeger, K.Hyeon-Deuk, O.V.Prezhdo, Acc.Chem. Res., 46, 1280 (2013).

5. P.M.Zimmerman, C.B.Musgrave, M.Head-Gordon, Acc. Chem. Res., 46, 1339 (2013).

6. M.B.Smith, J.Michl, Annu.Rev.Phys.Chem., 64, 361 (2013)

7. X.Feng, A.V.Luzanov, A.I.Krylov, J.Phys. Chem. Lett., 4, 3845 (2013).

8. A.V.Luzanov, Uspekhi Khimii, 49, 1033 (1980).

9. A.V.Luzanov, O.A.Zhikol, Int.J.Quant. Chem., 110, 902 (2010). 
10. A.V.Luzanov, O.A.Zhikol, in: Practical Aspects of Computational Chemistry I: An Overview of the Last Two Decades and Current Trends, eds. J.Leszczynski, M.K.Shukla, Springer, New York (2012), p.415.

11. F.Plasser, H.Lischka, J.Chem. Theory Comp., 8, 2777 (2012).

12. I.Shavitt, R.J.Bartlett, Many-body Methods in Chemistry and Physics, Cambridge University Press, Cambridge (2009).

13. A.V.Luzanov, V.F.Pedash, Theor.Exp.Chem., 15, 338 (1979)
14. A.I.Krylov, Annu.Rev.Phys.Chem., 59, 433 (2008).

15. A.B.Zakharov, V.V.Ivanov, L.Adamowicz, $J$. Phys. Chem. C, 118, 8111 (2014).

16. T.Yasuike, K.Nobusada, M.Hayashi, Phys. Rev.A, 83, 013201 (2011).

17. A.V.Luzanov, Int.J.Quant. Chem., 112, 2915 (2012).

18. A.I.Krylov, C.D.Sherrill, M.Head-Gordon, $J$. Chem. Phys., 113, 6509 (2000). 